Special Issue of the 8th International Advances in Applied Physics and Materials Science Congress (APMAS 2018)

\title{
Characterization of Polyaniline/Single-Walled Carbon Nanotube Composite Films Prepared by Plasma Polymerization
}

\author{
A.S. WASFi* AND H.O. ISMAEL \\ Physics Department, College of Science, University of Baghdad, Baghdad, Iraq
}

\begin{abstract}
Polyaniline (PANI)/single-walled carbon nanotube (SWCNT) composite films with good uniformity were prepared by in situ plasma polymerization of aniline containing SWCNTs. A unique method was used to inject the monomer and SWCNTs simultaneously into the plasma reactor. Chemically pre-functionalized SWCNTs-COOH and pristine SWCNTs were used in this study. Both types of the SWCNTs were plasma functionalized during their path to the substrate through the microwave plasma, which led to a uniform dispersion within the PANI matrix. The structure and morphology of the films were characterized by the Fourier transform infrared, X-ray diffraction, atomic force microscopy, and scanning electron microscopy. The Fourier transform infrared method confirmed the chemical bonding and the formation of the nanocomposite. While the X-ray diffraction results indicated that the composite has a relatively high ordered crystalline structure, which is expected to reveal high electrical conductivity. The atomic force microscopy micrographs specified a uniform distribution of the SWCNTs in PANI matrix with no aggregations and low roughness average, whereas the scanning electron microscopy micrographs revealed that SWCNTs of both kinds were completely coated by PANI and well dispersed in a continuous polymer matrix forming a conductive network. Films conductivity was examined by the Hall effect; the PANI/SWCNT composite showed a conductivity of $25 \mathrm{~S} / \mathrm{cm}$ with using pristine SWCNTs of $1.5 \mathrm{wt} \%$ and $50 \mathrm{~S} / \mathrm{cm}$ for composite using chemically pre functionalized SWCNTs-COOH of the same loading percentage, compared to that of pure PANI of $10^{-8} \mathrm{~S} / \mathrm{cm}$. It is concluded that the improved conductivity of the PANI/SWCNT composite films is due to the strong interaction between SWNTs and PANI, which simplifies the effective degree of electron delocalization.
\end{abstract}

DOI: 10.12693/APhysPolA.135.578

PACS/topics: polyaniline composite, single-walled carbon nanotubes, plasma polymerization, thin films, electrical conductivity

\section{Introduction}

Plasma polymerization is a solvent-free and worthwhile technique for producing organic films of different thicknesses on a diverse family of substrates from nearly any organic vapors. The chemical and physical structures of the plasma polymerized thin films are unlike those produced by wet processing methods. For instance, the chemical and electrochemical ones. The parameters which powerfully affect the molecular structure and properties of the plasma polymer are the monomer itself and its flow rate, reactor pressure, gas phase composition, and the geometry of the reactor [1]. Plasma polymerization is the best technique to get a very thin, uniform, and pinhole-free film. The films formed by plasma polymerization are insoluble, chemically inert, and thermally stable [2]. PANI is considered to be one of the most multipurpose conducting polymers with an extensive spectrum of tunable properties [3]. Its structural characteristics can be varied by changing different synthesis parameters and additives. The in situ polymerization of aniline monomer in the existence of carbon nanotubes (CNTs) is one of the most efficient and versatile ways to synthesize PANI nanocomposites. Due to their good interfacial

*corresponding author; e-mail: a.wasfi@scbaghdad.edu.iq adhesion with PANI matrix, CNTs are essential for successful preparation, and they have enhanced optical and electrical properties compared with base polyaniline [4].

In this work, a different approach to the improvement of PANI/CNT composite films with well-developed surface morphology is presented. It consists of using microwave plasma polymerization to produce thin films that are unlike the films produced by conventional methods. The monomer with dispersed CNTs was directly injected into the plasma environment induced by microwave discharge. The reactor was designed to deposit films at conditions near to chemical reaction energetics. By means of this arrangement, glass substrates were coated with a PANI/SWCNT composite film. The structural, morphological, and electrical characterizations exhibited the detailed features of the composites and their improved electrical conduction.

\section{Experimental procedure}

\subsection{Materials}

SWCNTs (purity 95\%) were supplied by neutrino factory, India. They have a diameter of 1-2 $\mathrm{nm}$ and a length about $30 \mu \mathrm{m}$. Chemically pre-functionalized SWCNTs$\mathrm{COOH}$ and pristine SWCNTs were used in this study. The aniline (purity 99.99\%) was supplied from Hopkin and Williams Ltd., England. 


\subsection{Synthesis of PANI/SWCNT composite films}

The microwave plasma polymerization reactor used in this work and the procedure of film deposition have been described in a previous publication [5]. The aniline precursor and the SWCNTs with different loading percentage, after being dispersed ultrasonically in aniline for $30 \mathrm{~min}$, were injected at the same time into the plasma reactor through a pulsed atomizer (car fuel injector) that can maintain a good vacuum separation between the pressurized aniline reservoir and the evacuated plasma reactor. The thickness of the deposited films depends on the amount of the injected monomer in the Ar plasma as a spray pulse and particularly on the loading percentage of the CNTs. The control of the spray pulse duration and its recurrence with time was necessary to keep the pressure in the chamber within the plasma working pressure range of $0.1-1.0$ mbar. When a pulse of the monomer comes into the evacuated reactor, it will directly vaporize by flash boiling in the plasma environment and when the habitation period of the vapor in the plasma was properly selected, it was predictable to have great radical percentage nearby the substrate. The plasma-induced polymerization of aniline vapor with SWCNTs was composed of two simultaneous processes that were the surface activation (functionalization) of the SWCNTs and polymerization of the aniline. The monomer was adsorbed on the surface of the SWCNTs due to their reactivity and high surface area, and then through their movement in the plasma environment, the polymerization begins on the CNTs and persists in the pathway of the substrate. Therefore, the film was expected to have plasma functionalized SWCNTs with PANI, chemically bonded, inserted in the PANI matrix. The glass substrate, after the suitable cleaning process, was fixed inside the reactor at a position that was exposed to the plasma having a uniform electron density of $10^{17} \mathrm{~cm}^{-3}[6]$.

\section{Results and discussions}

\subsection{FTIR measurements}

The Fourier transform infrared (FTIR) spectra for PANI and its composites in the wavelength range of 400$4000 \mathrm{~cm}^{-1}$ are presented in Fig. 1. They show overlaid influences owing to the chemical bonding among the dangling bonds at the CNT surface and polyaniline. For pure PANI, the peak at $\approx 3410 \mathrm{~cm}^{-1}$ specifies the $\mathrm{N}-\mathrm{H}$ stretching of the aromatic ring in PANI and its composite, whereas the feature peaks at $\approx 1527 \mathrm{~cm}^{-1}$ and $\approx 1640 \mathrm{~cm}^{-1}$ are due to the $\mathrm{C}=\mathrm{C}$ asymmetric and symmetric stretching of benzenoid and quinoid rings, respectively, which are characteristic to protonated polyaniline. The peaks at 1269 and $1176 \mathrm{~cm}^{-1}$ are ascribed to the $\mathrm{C}-\mathrm{N}$ stretching vibration of the secondary aromatic amine group, and the peak at $1072 \mathrm{~cm}^{-1}$ are assigned to aromatic $\mathrm{C}-\mathrm{H}$ in-plane bending vibration. The peak at $752 \mathrm{~cm}^{-1}$ represents the $\mathrm{C}-\mathrm{H}$ out of plane bending vibration [7-10]. The FTIR spectra of both composites

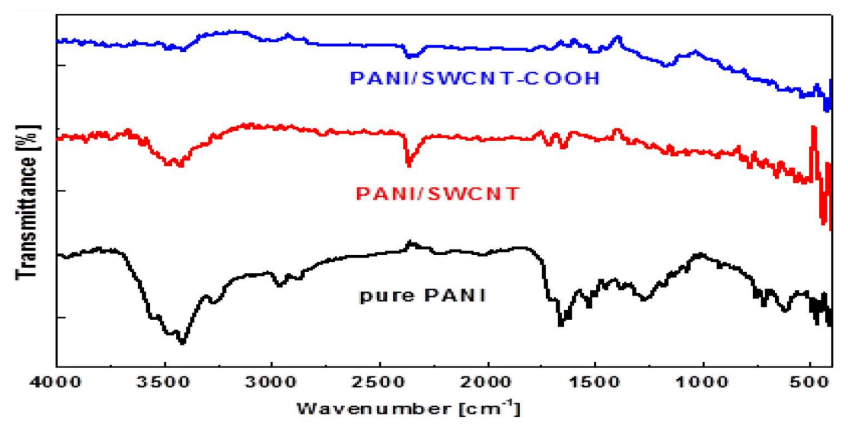

Fig. 1. FTIR spectra for PANI and PANI/SWCNT composite films of $1.5 \mathrm{wt} \%$ loading percentage of SWCNTs.

that used the pristine SWCNT with PANI and the chemically pre-functionalized SWCNT-COOH with PANI are almost near to that of pure PANI with some shift. The peaks intensities decreased, indicating the interaction between PANI chains and the surfaces of the CNTs forming $\pi-\pi$ non-covalent bonds. The additional peaks observed at 1126 and $1172 \mathrm{~cm}^{-1}$ are definite for $\mathrm{CC},-\mathrm{C}-\mathrm{NH}$ aromatic rings, respectively. This is the major characteristic which confirms that PANI-chains are bonded to CNTs surfaces. The peak at $\approx 1126 \mathrm{~cm}^{-1}$ is a measure of the degree of electron delocalization and consequently, it is the feature peak of PANI electrical conductivity [11, 12]. The intensity of this peak increases with the addition of CNTs, which agrees well with our increased electrical conductivity measurements.

\subsection{X-ray diffraction}

Figure 2 shows the XRD patterns of PANI, PANI/SWCNT-COOH composite in addition to PANI/SWCNT composite. For pure PANI, crystalline peaks appear at $10.74^{\circ}, 15.82^{\circ}$, and $24.44^{\circ}$, which can be assigned to the (011), (011), and (200) reflections from crystal planes of PANI, respectively [13]. The X-ray patterns of PANI/SWCNT-COOH and PANI/SWCNT composites show the typical peaks of PANI and SWCNTs, indicating the existence of SWCNTs in the polymer matrix. Compared with PANI X-ray pattern, the intensity of the peak at $24.44^{\circ}$ has increased and shifted

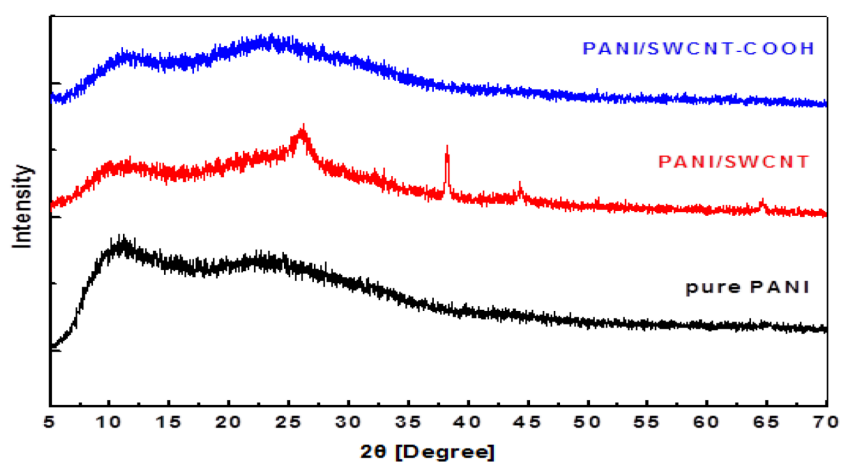

Fig. 2. XRD for PANI and PANI/SWCNT composite films of $1.5 \mathrm{wt} \%$ loading percentage of SWCNTs. 
to $26^{\circ}$, which indicates that this peak is mainly due to the overlapping of PANI and SWCNTs. It also shows that the composite films have a relatively highly ordered crystalline structure, which improves the crystallinity of the composite considerably, and is expected to reveal high electrical conductivity. The decrease in peak heights of the PANI/SWCNT-COOH composite pattern might be caused by the better polymer coating of the SWCNTs-COOH surface in the polymerization process due to their higher degree of functionalization [14].

\subsection{AFM measurements}

The surface topography of PANI film Fig. 3a, as evidenced by AFM, shows a continuous and smooth surface with a roughness average of $0.38 \mathrm{~nm}$. While the micrographs of PANI/SWNT and PANI/SWCNT-COOH composite films, Fig. 3b and c indicates a uniform distribution of SWCNTs in PANI matrix with no aggregations and roughness average of $6.99 \mathrm{~nm}$ and $2.26 \mathrm{~nm}$, respectively. All the CNTs are coated with PANI forming a continuous matrix which encloses the nanocarbon tubes in small cages. It is clear that the roughness average of the PANI/SWCNT-COOH composite film is less than that of PANI/SWCNT composite film. This may be due to the much more bonds that can be formed on the surface of the SWCNTs-COOH than those formed on the surface of the pristine SWCNTs with less functionalization opportunity, which are plasma functionalized only, due to the short exposure time to the Ar plasma. This would hearten PANI to cover the tubes more effectively thus filling most of the voids at the film surface.

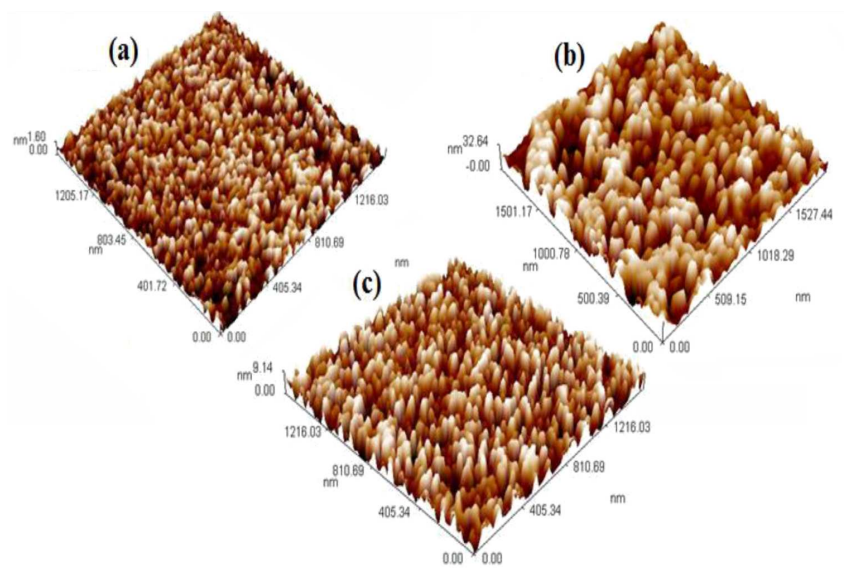

Fig. 3. (a) AFM topography of PANI film, (b) PANI/SWCNT composite film of $1.5 \mathrm{wt} \%$ of pristine SWCNTs, and (c) PANI/SWCNT-COOH composite film of $1.5 \mathrm{wt} \%$ of chemically pre-functionalized SWCNTs.

\subsection{Scanning electron microscope imaging}

The scanning electron microscope (SEM) micrographs of films deposited under the same deposition conditions are presented in Fig. 4. Micrograph of pure PANI film showed that it has a continuous, smooth, and tidy surface as in Fig. 4a. The micrographs in Fig. 4b and $\mathrm{c}$ are for PANI/SWCNT and PANI/SWCNT-COOH composite films of $1.5 \mathrm{wt} \%$ of both SWCNTs and SWCNTs$\mathrm{COOH}$ loading percentage, respectively. These figures
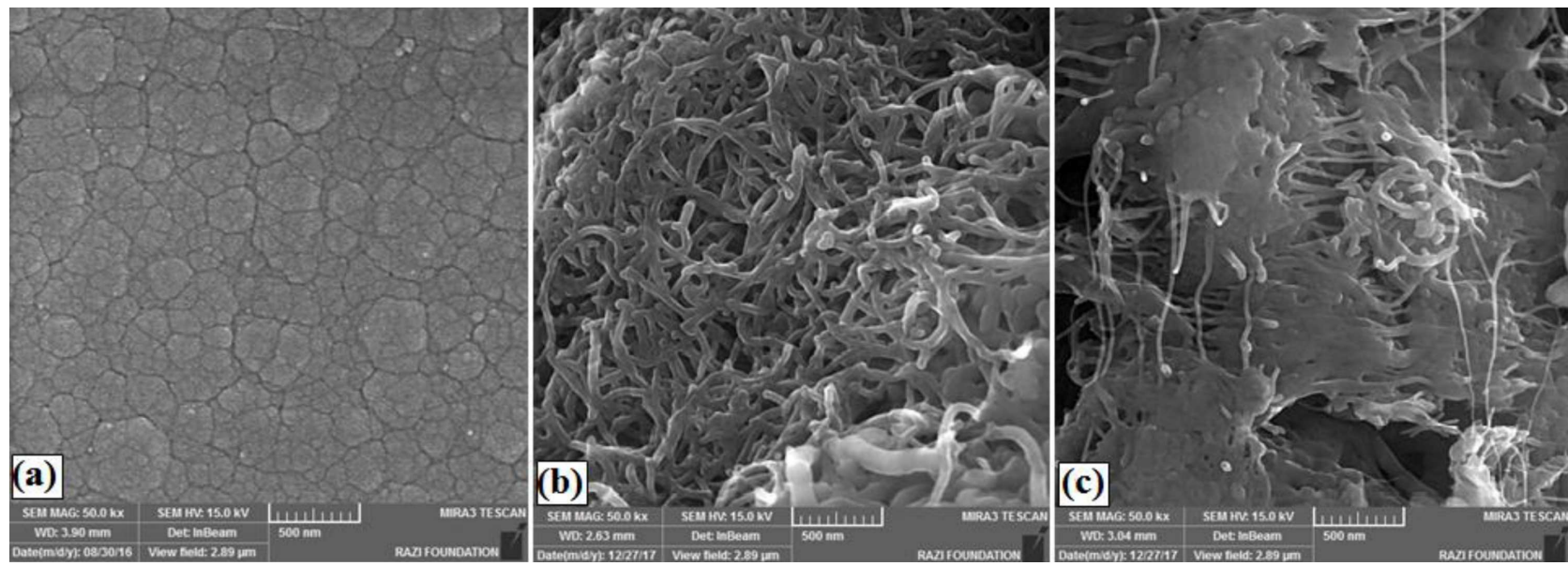

Fig. 4. SEM micrographs of films deposited under the same conditions: (a) PANI film, (b) PANI/SWCNT composite film, and (c) PANI/SWCNT-COOH composite film.

show a high density of embedded SWCNTs very well dispersed in a continuous polymer matrix forming a conductive network. The SWCNTs themselves are smooth and with diameters of 1-2 nm. However, when they were introduced into the reactor with the aniline, tubes smooth surfaces changed into a rough structure due to the plasma polymerization of aniline that was adsorbed by the tubes and their diameters were enhanced to 50-100 nm. It can be noticed that PANI agglomerated on the CNTs forming islands that were connected by the CNTs in the PANI/SWCNT-COOH composite film. While PANI coated the CNTs uniformly forming a core-shell structure and almost no agglomeration of PANI was observed in the PANI/SWCNT composite film. 
This may be due to the high degree of functionalization of the SWCNTs-COOH, which is chemically prefunctionalized in addition to the plasma functionalization, in comparison with pristine SWCNTs that received a plasma functionalization only. This has led to an increase in the interfacial bonding between the CNTs and the polymer that stimulated the enormous growth of PANI.

\subsection{Electrical conductivity measurements}

The electrical conductivity of PANI and PANI/SWCNT composite films of both SWCNTs types and different loading percentages were examined by the Hall effect. The results are shown in Table I and Fig. 5. It is clear that the conductivity is the lowest for PANI films and increases with the loading percentage of CNTs in the film. It increases slowly for up to $0.1 \mathrm{wt} \%$ CNTs loading percentage of both types in the composite and increases rapidly beyond $0.1 \mathrm{wt} \%$ CNTs. This increase in connectivity can be ascribed to the mutual influence among PANI and CNTs

TABLE I

The electrical parameters of PANI and PANI/CNT composite thin films.

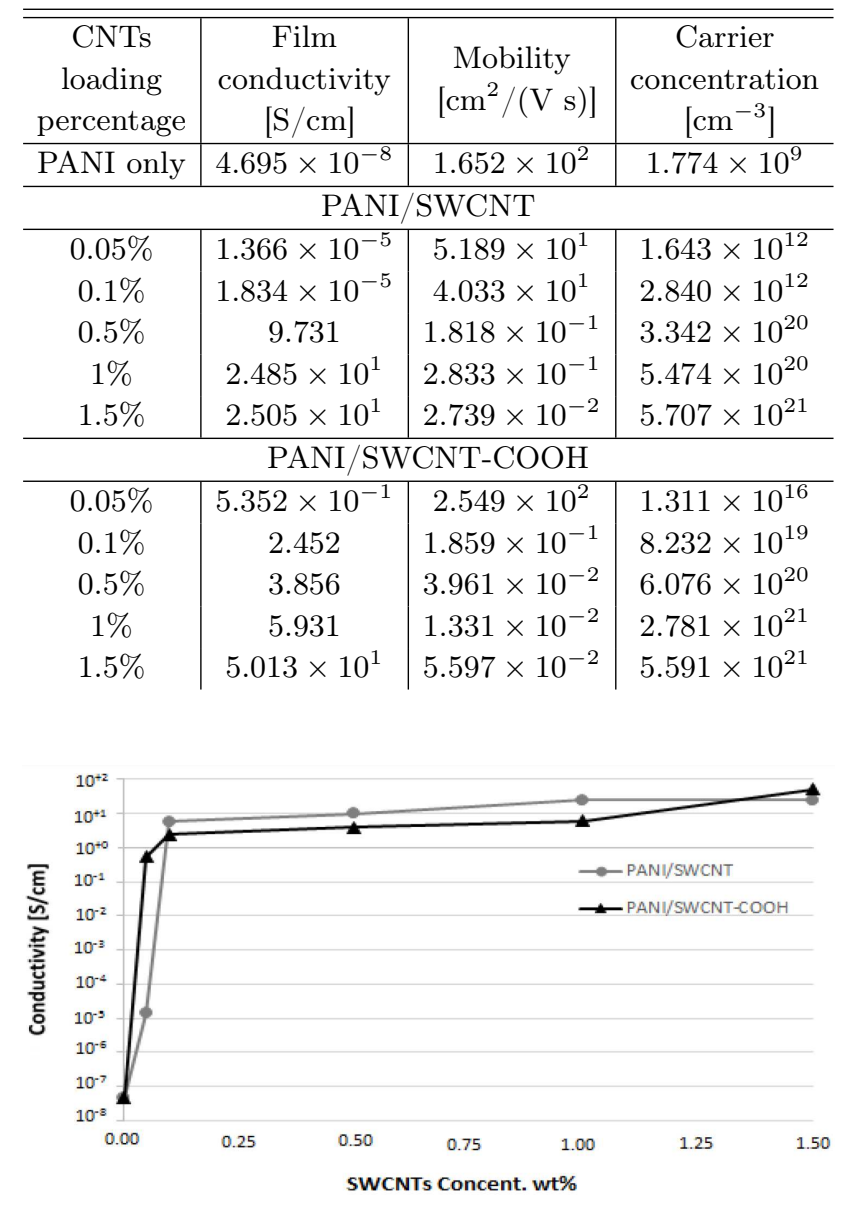

Fig. 5. The electrical conductivity of PANI/SWCNT films as a function of the SWCNTs loading percentage. in creating an interpenetrating conductive grid. The less conductivity at fewer CNTs loading percentage is owing to too much PANI made accumulation above the CNTs. Therefore, conductive channels created by CNTs are detached from each other. This prohibited the transfer of active charge through the conductive grid. Hence, the nanocomposites conductivity is attributed to pure PANI only. As the loading percentage of CNTs increased, they got in touch with each other creating a three-dimensional grid where the conductivity is abruptly recognized. The loading percentage of CNTs at which this evolution in conductivity happens is identified as the percolation onset. PANI/SWCNT composite film of $1.5 \mathrm{wt} \%$ loading percentage SWCNTs showed a conductivity of $25 \mathrm{~S} / \mathrm{cm}$, which is almost nine orders of magnitude greater than that observed for pure PANI film, while the conductivity of PANI/SWCNT-COOH composite of the same loading percentage and deposition condition is the highest, that is $50 \mathrm{~S} / \mathrm{cm}$. This is attributed to the high degree of functionalization accomplished in the SWCNTs-COOH, which causes an increase in the interfacial bonding between the CNTs and the polymer matrix leading to a better mutual interaction with the polymer matrix and an effective increase in the conductivity.

\section{Conclusion}

In situ microwave plasma polymerization is considered to be an active technique to intentionally improve the nanotubes dispersion and interaction with the polymer matrix. It allows covering of the convex walls of the nanotubes by the polymer macromolecules. This then offers a better nanotubes dispersion and development of a robust interface between the nanotube and the polymer matrix. PANI/SWCNT composite films, with different functionalization conditions of SWCNTs, were synthesized by microwave plasma polymerization. The FTIR spectra show the effective structural modification and the establishment of PANI/SWCNT composites. The incorporation of the plasma functionalized SWCNTs of both types into the PANI matrix improved the transport properties of the nanocomposite. It is clear that the SWCNTs-COOH of chemical pre-functionalization and plasma functionalization provide a higher composite conductivity, which is a crucial matter for some scientific applications.

\section{References}

[1] O. Kylián, A. Choukourov, H. Biederman, Thin Solid Films 548, 1 (2013).

[2] N. Alissawi, T. Peter, T. Strunskus, C. Ebbert, G. Grundmeier, F. Faupel, J. Nanopart. Res. 15, 1 (2013)

[3] G.B.V.S. Lakshmi, A. Dhillon, A.M. Siddiqui, M. Zulfequar, D.K. Avasthi, Europ. Polym. J. 45, 2873 (2009).

[4] W. Chang, C. Wang, C. Chen, Electrochim. Acta 212, 130 (2016). 
[5] A.S. Wasfi, H.R. Humud, M.A. Abed, M.E. Ismael, Int. J. Chem. Tech. Res. 10, 886 (2017).

[6] A.S. Wasfi, H.R. Humud, M.E. Ismail, Iraqi J. Phys. 13, 1 (2015).

[7] Y. Li, H. Peng, G. Li, K. Chen, Europ. Polym. J. 48, 1406 (2012).

[8] G. Socrates, Infrared and Raman Characteristic Group Frequencies, 3rd ed., Wiley, England 2001.

[9] M.V. Kulkarni, B.B. Kale, Sens. Actuat. B Chem. 187, 407 (2013).
[10] A. Kausar, Am. J. Polym. Sci. Eng. 3, 149 (2015).

[11] J. Yang, X. Wang, X. Wang, R. Jia, J. Huang, J. Phys. Chem. Solids 71, 448 (2010).

[12] A.K. Sharma, G. Chaudhary, I. Kaushal, U. Bhardwaj, A. Mishra, Am. J. Polym. Sci. 5, 1 (2015).

[13] L. Ding, Q. Li, D. Zhou, H. Cui, H. An, J. Zhai, J. Electroanal. Chem. 668, 44 (2012).

[14] V.H. Nguyen, J.J. Shim, J. Spectrosc. 2015, 1 (2015). 